\title{
Method for Realization of Number Estimation Subsystem of Netted Radar Emitter Signals
}

\author{
Cheng Xu-de, Xu Bing, Wang Hong-li, Liu Wei \\ Dept. of 5, Wuhan Mechanical Technology, Wuhan 430075, China \\ email:1712178221@qq.com.cn
}

Keywords: Cyclic correlation; Netted radar; LFM; Estimation of source number

\begin{abstract}
Based on the modern battlefield electronic reconnaissance, a realization method of netted radar signal sources number estimation subsystem is presented. The cyclic correlation function of linear FM signal is deduced in this paper, and the function is used to estimate the number of signal sources. This cyclic correlation based method is computationally efficient and easy to realize due to the utilization of FFT (Fast Fourier Transform) in calculating the cyclic correlation function. Simulation experiments are provided for evaluating the estimation performance, and experimental results have proved the validity of the method.
\end{abstract}

\section{Foreword}

In modern battlefield, there're vast amount of emitters in dense distribution, which cause overloaded radar receiver with enormous data interception, and even a lengthy period if such massive data are sifted and compared one by one with classic radar signal sorting algorithm. Many scholars turn to apply blind signal separation (BSS) technology to radar signal sorting. The principle of BSS technology is to realize the recovery of source signal's waveform through optimum estimate of hybrid matrix ${ }^{[1]}$, so as to extract the characteristics of the recovered waveform and derive the information related to radar signal. In the process of electronic reconnaissance, signals intercepted usually contain radar signal, clutter and noise, thus these signals need to be processed before feeding in blind signal sorting module. Determination of number of signal sources makes available priori information for BSS algorithm, and the precision of number determined has direct impact on that of blind signal sorting, which is the key part in the whole sorting process ${ }^{[3]}$. In practical application, the number of observation signals is unknown, which is only obtained by means of source number estimation algorithm. In this dissertation, in the context of battlefield electronic reconnaissance, the author suggests the realization method for number estimation subsystem of netted radar emitter signals applied to radar electronic countermeasures system to estimate the number of netted radar emitter signals on the principle of cyclic correlation property.

\section{Principle for Number Estimation of Signals}

In modern war, the two parties involved in radar electronic countermeasure are fighter plane and netted radar. In the radar network, radars of different properties emit radar signals to the same airspace for the purpose of reconnaissance therein. Assume that there're $n$ radars in the radar network but at different geographic orientations, in this system, this characteristic is represented by information on the angle of each radar emitter signal, as well as attenuation condition of such information fed in radar warning receiver (RWR). Emitter signals from $n$ radars are expressed as follows:

$$
s(n)=\left[\mathrm{s}_{1}(n), \mathrm{s}_{2}(n) \ldots, \mathrm{s}_{\mathrm{n}}(n)\right]^{\mathrm{T}}
$$

Assume that there're $m$ receiving antenna with RWR on electronic scout for intercepting radar signals, in the case radar emitter signals are intercepted by these receiving antenna, signal intercepted by antenna $i$ can be expressed as follows:

$$
x_{i}(n)=\sum_{k=1}^{N} b_{i k} s_{k}(n) \mathrm{e}^{-j 2 \pi f_{k} \tau_{i k}}
$$


Where: $b_{i k}$ and $\tau_{i k}$ respectively mean the amplitude attenuation and time delay when the signal is intercepted by antennai; $f_{k}$ means frequency of signal $s_{k}(n)$; the emitters can be estimated from signals received by the antenna on electronic scout. It's nearly impossible to estimate number of radar emitters without priori knowledge, to this end, assumptions are made herein to allow estimation of number of radar emitters in this context by means of cyclic correlation property, as follows:

Assumption 1: radar emitter signals are independent with each other;

Assumption 2: Target signals are subject to non-Gaussian distribution;

Assumption 3: Target signals are of different cyclic frequency from that of other emitter signals.

Above assumptions are made based upon actual condition. Due to different properties of various radars in radar network, emitter signals are different in terms of such factors as amplitude, waveform, frequency, etc. For emitter signals of various radars, such signals can be regarded as independent with each other.

\section{Cyclic Correlation Function}

Signals intercepted by RWR are expressed as follows:

$$
x(t)=s(t)+n(t)
$$

Where: $s(t)=$ emitter signal, $n(t)=$ noise signal; take mean of received signals to derive:

$$
M_{x}(t)=E[x(t)]
$$

Obviously, mean of the signals are the function of time, it's unable to estimate mean of the signals direct from mean time, thus mean of samples is used to estimate statistic mean, then:

$$
M_{x}(t)=E[x(t+n T)]=\lim _{N \rightarrow \infty} \frac{1}{2 N+1} \sum_{n=-N}^{N} x(t+n T)
$$

Where: $T$ =signal cycle, it's obvious that $M_{x}(t)$ is the function of cycle $T$. Expand statistic mean to Fourier series to derive:

$$
M_{\chi}(t)=\sum_{m=-\infty}^{+\infty} M_{x}^{m / T} e^{j 2 \pi m t / T}
$$

Corresponding Fourier coefficient is:

$$
M_{x}^{m / T}=\frac{1}{T} \int_{-T / 2}^{T / 2} M_{x}(t) e^{-j 2 \pi m t / T} d t=\left\langle x(t) e^{-j 2 \pi m t / T}\right\rangle_{t}
$$

Where: \langle\rangle$_{t}=$ time mean. In common form, the coefficient can be expressed as follows:

$$
M_{x}^{\varepsilon}(t)=\left\langle x(t) e^{-j 2 \pi \varepsilon t}\right\rangle_{t}
$$

Where: $\varepsilon$ =harmonic frequency. Generally, $\varepsilon$ frequency component $M_{x}^{\varepsilon}(t)$ of time-varying mean $M_{x}(t)$ is called cyclic mean, and $\varepsilon$ is called first order cyclic frequency.

It's obvious from equation (8) that cyclic mean is equivalent to time mean after left shifting of frequency $\varepsilon$ with spectrum of received signals $x(t)$. Thus, as long as frequency $\varepsilon$ line exists in power spectrum of the signals, i.e. $M_{x}^{\varepsilon} \neq 0$, then such signals are stable during first order cycle. However, there're some unstable signals lacking of first order cycle stability, but these unstable signals are usually with more pinpoint cyclic property, which is called cycle stability property. Then, cyclic correlation function is defined. Considering time-varying correlation function with this type of unstable signals, then:

$$
R_{x}(t ; \tau)=E\left[x(t+\tau / 2) x^{*}(t-\tau / 2)\right]
$$

Inverse equation (9) with above conversion method, then:

$$
R_{x}(t ; \tau)=\lim _{N \rightarrow \infty} \frac{1}{2 N+1} \sum_{n=-N}^{N} x\left(t+n T_{0}\right) x^{*}\left(t+n T_{0}-\tau\right)
$$

Expand to Fourier series, and corresponding Fourier coefficient is:

$$
R_{x}^{\varepsilon}(\tau)=\left\langle x(t+\tau / 2) x^{*}(t-\tau / 2) e^{-j 2 \pi t}\right\rangle_{t}
$$


Then, $R_{x}^{\varepsilon}(\tau)$ is defined as cyclic autocorrelation function; However, for radar emitter signal ${ }^{[2]}$ :

$$
s(t)=\operatorname{rect}(t / T) \exp \left[j 2 \pi\left(f_{0} t+\frac{1}{2} k t^{2}\right)\right]
$$

Its cyclic correlation function is:

$$
R_{x}^{\varepsilon}(\tau)=\lim _{T \rightarrow \infty} \frac{1}{T} \int_{-T / 2}^{T / 2} x(t+\tau / 2) x^{*}(t-\tau / 2) e^{-j 2 \pi \varepsilon t} d t=2 \pi\left[\delta(2 \pi \varepsilon)+R_{n}(\tau) \delta(\varepsilon)\right]
$$

\section{Method for Number Estimation of Emitters}

Presently, mature methods available for number estimation of radar emitters include information theoretic criterion based algorithm, Gerschgorin's disk estimation (GDE) theory, singular value decomposition (SVD) and etc ${ }^{[5]}$. However, most of these methods are conducted under over-determined or positive definite experimental condition. It's obvious that, in the context herein, number of netted radar should be more than that of receiving antenna on electronic scout; In actual situation, radar network generally consists of multiple radars of different properties and functions, and basically 4-6 receiving antenna are sued for RWR on a modern mainstream electronic scout. The background condition is large amount of radar emitters and small amount of receiving antenna, namely, underdetermined condition, under which foresaid classic method for number estimation of emitters is poor in effect, thus number of emitters is estimated on the principle of cyclic correlation property of radar emitter signals.

Received signal $X(t)$ is expressed in the form of vector, as follows:

$$
X(t)=\left[x_{1}(t), x_{2}(t), \ldots x_{n}(t)\right]^{T}
$$

The model of received signal is expressed as follows:

$$
X(t)=A S(t)+N(t)
$$

As noise signal is subject to Gaussian distribution and uncorrelated to incident signal, as assumed above, then, take cyclic correlation on both sides of equation (15) for SVD conversion, then:

$$
R_{X X}^{(\varepsilon)}(\tau)=U S V^{H}
$$

Where: unitary matrixes $U$ and $V$ are respectively left singular matrix and right singular matrix; $S$ is diagonal matrix, diagonal element is singular value of $R_{X X}^{(\varepsilon)}(\tau)$. As the order of cyclic correlation matrix of observation signal is equal to the order of cyclic correlation matrix of emitter signal, corresponding unitary matrixes are partitioned from descending order of singular values, then:

$$
U=\left[\begin{array}{ll}
U_{s} & U_{n}
\end{array}\right] \quad V=\left[\begin{array}{ll}
V_{s} & V_{n}
\end{array}\right]
$$

Obviously, after partitioning of unitary matrixes $U$ and $V$, subspace from spanning of $U_{s}$ or $V_{s}$ is called signal subspace; while subspace from spanning of $U_{n}$ or $V_{n}$ is called noise subspace; projection of cyclic correlation function of some signals in noise subspace is zero, namely:

$$
R_{X X}^{(\varepsilon)}(\tau) U_{n}=0 \quad A R_{S S}^{(\varepsilon)}(\tau) A^{H} U_{n}=0
$$

In equation (19), it represents inner product of signal vector and noise matrix, which is zero only when signal vector and noise matrix are normal; due to space spectrum of the signals is as follows:

$$
P=1 / a\left(\omega_{i}\right)^{H}\left(U_{n}\right)^{H} a\left(\omega_{i}\right)
$$

Therefore, only number of minimum values of $a\left(\omega_{i}\right)^{H}\left(U_{n}\right)^{H} a\left(\omega_{i}\right)$ needs to be derived, i.e. number of emitters included in observation signal. To conclude, procedures with regard to the method for number estimation of radar emitter signals based on cyclic correlation property are as follows: derive observation signal $X(t)$; take cyclic correlation function $R_{X X}^{(\varepsilon)}(\tau)$ for observation signal; derive and array singular value of cyclic correlation matrix by means of SVD; partition corresponding unitary matrixes after array to divide into signal subspace and noise subspace; derive number of minimum values of $a\left(\omega_{i}\right)^{H}\left(U_{n}\right)^{H} a\left(\omega_{i}\right)$, then number of emitter signals is derived. 


\section{Analysis of System Test and Simulation Experiment Results}

In this section, SystemVue software is applied to simulation experiment. SystemVue software is developed by Agilent Technologies, which include in-built particular radar and electronic battle modules, complete radar electronic countermeasures system in pure software format can be formed with SystemVue. Alternatively, corresponding hardware equipments can be interconnected via interfaces to realize semi-physical system. In addition, this software functions to load Matlab application to form program package, or make direct programming with relevant language. For this time, SystemVue is used in simulation to realize radar signal estimation subsystem.

In the simulation, 5 radar emitter signals are assumed. Radar signals are projected on receiving antenna at $-42^{\circ},-12^{\circ}, 22^{\circ}, 55^{\circ}$ and $59^{\circ}$ incidence angles respectively. Mixed radar signals after transmission can be realized by setting of amplifier with various magnification times after generating modules of radar signals.

Due to different distances between radar and receiver, airborne attenuation level varies accordingly, thus distance between radar and receiver can be simulated by adjusting various magnification times of amplifier. Particular distance can be calculated by basic equation of radar.

Radar signals are set as linear frequency modulation signals, all signals are in the form of $s_{n}(t)=A e^{j \frac{k t^{2}}{2}+j f_{0} t}$, specific parameter for each signal is described by initial frequency and modulation slope, as listed in Table 1:

Table 1. Radar Signal Parameters

\begin{tabular}{ccc}
\hline No. & Initial Frequency & Modulation Slope \\
\hline 1 & 800 & 3 \\
2 & 1000 & 5 \\
3 & 1200 & 7 \\
4 & 1400 & 9 \\
5 & 1600 & 11 \\
\hline
\end{tabular}

Experiment 1: Effect of SNR to estimation performance experiment. Assume there're 3 receiving antenna, increase SNR from 0dB to 20dB, conduct Monte Carlo analysis for 100 times at each SNR, the results are as shown in Chart 2. It is seen from Chart 2 that, estimation correctness of the algorithm is low at low SNR, and it's unable to correctly estimate number of emitter signals; however, when SNR increases, correctness ratio of cyclic correlation algorithm ultimately approaches 1 , indicating this method contributes to correct number estimation of netted radar emitter signals.

Experiment 2: Effect of number of receiving antenna to estimation performance. Conduct tests respectively with 3, 4 and 5 receiving antenna at SNR ranging from $0 \mathrm{~dB}$ to $20 \mathrm{~dB}$, then conduct Monte Carlo analysis for 100 times at each SNR, the results are as shown in Chart 3.

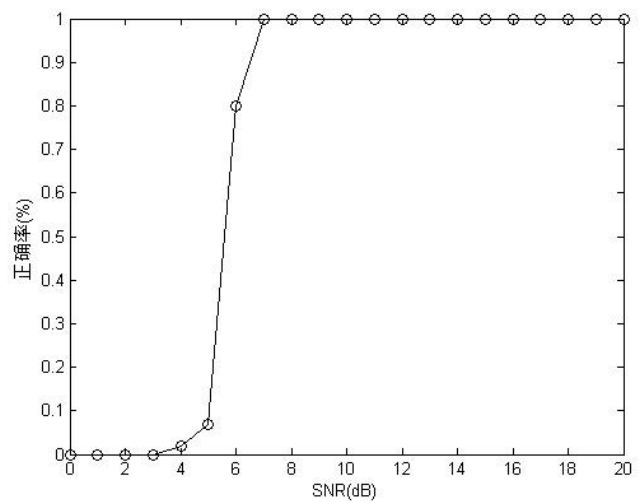

Chart 2. Effect of SNR to Estimation Performance

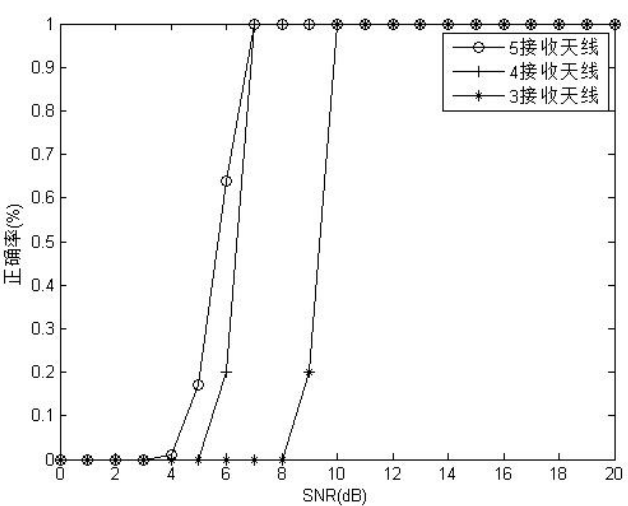

Chart 3. Effect of Number of Receiving Antenna to Estimation Performance 
It's seen from Chart 3 that, different numbers of receiving antenna have no impact on ultimate estimation correction, and all estimations are correct. However, more receiving antenna contributes to better estimation at low SNR, indicating more receiving antenna contributes to better adaptability.

\section{Conclusion}

This dissertation expatiated in the context of electronic reconnaissance in modern battlefield to realize the number estimation subsystem of netted radar emitter signals, which has been applied to radar electronic countermeasures system. In addition, desirable stability is proved in number estimation of mixed linear frequency modulation signals of unknown number by the method of cyclic correlation estimation suggested herein, making it valuable in practical application. Further work should be focused upon the problem of number estimation of signals at low SNR, this is because that, in radar electronic countermeasure, radar emitter signals attenuate substantially in airspace, to the extent to be covered by noise when such signals are received by the receiver, which lead to tremendous difficulty for estimation, and the author envisages to make further study with stochastic resonance theory.

\section{References}

[1] FAN Yun, WANG Xiang, HUANG Zhitao, ZHOU Yiyu. A Blind Signal Extraction Algorithm Based on Cyclostationary Constraint[J]. Journal of Astronautics. 2012.07(07):978-983

[2] SHI Jianfeng, XIAO Lisheng, ZHU Liangxue Characteristic Parameter Estimation of Chirp Signal Based on Cyclic Spectrum Amplitude[J]. Electronic Warfare Technology. 2004.06(3):7-9

[3] ABHIJIT A. Shah, DONALD W. Tufts. Determination of the Dimension of a Signal Subplace from Short Data Records[C]. IEEE Transactions on Signal Processing. 1994.09(42): 2531-2535

[4] Tan Beihai, Zhao Min, Xie Sheng1i. Blind Separation Algorithms of BPSK Signals by Estimating Source Number[J]. Journal of Electronics\&Information Technology. 2009.07(07): 1624-1629

[5] HIROSHI Sawada, SHOKO Araki, SHOJI Makino. Underdetermined Convolutive Blind Source Separation via Frequency Bin-Wise Clustering and Permutation Alignment[C]. IEEE Transactions on Audio, Speech, and Language Processing. 2010.05:516-527

[6] S. Mavaddaty, A. Ebrahimzadeh. Blind Signals Separation with Genetic Algorithm and Particle Swarm Optimization Based on Mutual Information[J]. Radioelectronics and Communications Systems. 2011.09(54):315-324

[7] JIN Yan, JI Hongbing. A new cyclic autocorrelation based blind parameter estimation method for PSK signals[J]. JOURNA OF XIDIAN UNIVERSITY. 2006.12(33):892-895,901 